\title{
Alcohol Use Disorder Increases Risk of Traumatic Brain Injury-Related Hospitalization: Insights From 3.8 Million Children and Adolescent Inpatients
}

Noha Eskander ${ }^{1}$, Shruti Prabhudesai ${ }^{2}$, Hira Imran $^{3}$, Ozge Ceren Amuk ${ }^{4}$, Rikinkumar S. Patel ${ }^{5}$

1. Psychiatry, California Institute of Behavioral Neurosciences \& Psychology, Fairfield, USA 2. Psychiatry, Rajarshi Chhatrapati Shahu Maharaj Government Medical College, Kolhapur, IND 3. Medicine, Rawalpindi Medical University, Rawalpindi, PAK 4. Psychiatry, Koç University School of Medicine, Istanbul, TUR 5. Psychiatry, Griffin Memorial Hospital, Norman, USA

Corresponding author: Rikinkumar S.Patel, rpatel_09@arcadia.edu

\section{Abstract \\ Objectives}

We conducted a cross-sectional study to identify the demographic predictors of traumatic brain injury (TBI), and the risk of association of psychiatric comorbidities including alcohol use disorder (AUD) and TBI-related hospitalizations in the children and adolescent population.

\section{Methods}

We included 3,825,523 children and adolescent inpatients (age 8-18 years) using the nationwide inpatient sample (NIS) database (2010-2014), and 61,948 inpatients had a primary diagnosis of TBI. These inpatients were grouped by comorbid AUD $(\mathrm{N}=2,644)$. Multivariable logistic regression model adjusted for demographics, and psychiatric comorbidities including other substance use disorders (SUDs) was used to evaluate the odds ratio (OR) of AUD as a risk factor for TBI-related hospitalization.

\section{Results}

The majority of the TBI inpatients were adolescents (12-18 years, $82.2 \%)$, males (71.2\%), and whites (59.2\%). Males had three times higher odds (95\% CI 3.14-3.26) for TBI-related hospitalization compared to females. Among psychiatric comorbidities, mood (4.1\%) and anxiety (2.2\%) disorders were prevalent in TBI inpatients, and were not associated with increased odds for TBI-related hospitalization. Among SUD, alcohol and tobacco use (4.4\% each), and cannabis use (3.5\%) were prevalent, and among all substances, AUD was associated with higher odds (OR 3.5, 95\% CI 3.35-3.67) of TBI-related hospitalization. These patients with TBI and comorbid AUD also had higher odds for abusing stimulants (OR 5.11, 95\% CI 3.85-6.77), cannabis (OR 4.69, 95\% CI 4.12-5.34), and tobacco (OR 3.77, 95\% CI 3.34-4.27).

Received 05/27/2020

Review began 06/11/2020 Review ended 06/13/2020 Published 06/21/2020

\section{() Copyright 2020}

Eskander et al. This is an open access article distributed under the terms of the Creative Commons Attribution License CC-BY 4.0., which permits unrestricted use, distribution, and reproduction in any medium, provided the original author and source are credited.

\section{Conclusion}

AUD is an independent risk factor for TBI-related hospitalization with an increased risk of $50 \%$ in the children and adolescent population compared to non-alcohol users. TBI inpatients with AUD are prevalent in white, and male adolescents. These at-risk populations are also at higher risk of comorbid mood disorders and increased substance use including stimulants, cannabis, and tobacco.

Categories: Pediatrics, Psychiatry

Keywords: alcohol use, traumatic brain injury, concussion, alcohol dependence, substance use, alcohol misuse, children

\section{Introduction}

Traumatic brain injury (TBI) is the major cause of disability with about 145,000 children and adolescents suffer from permanent cognitive, behavioral, and physical effects. Half a million children under 14 years visit the ED due to TBI in the United States (US) [1]. The incidence of TBI is two times more common in boys and in children above 10 years. Motor vehicle accidents (MVAs) represent the leading cause of death in the age group five to 24 years, blacks present more commonly with TBI from MVA [2,3].

According to the 2018 National Survey on Drug Use and Health (NSDUH), an estimated 401,000 adolescents, that is, $1.6 \%$ of adolescents (12-17 years) had alcohol use disorder (AUD). Every year 1,825 young college students die from alcohol-related unintentional accidents [4]. AUD and TBI are bi-directionally linked, and AUD increases the risk of TBI. About $30 \%$ to $50 \%$ of patients treated for TBI were intoxicated at the time of injury. Binge alcohol drinking is a major risk factor for TBI, and vice versa there is a risk of alcohol abuse in 
post-TBI patients that worsens the prognosis and rehabilitation outcomes [5]. AUD leads to risk-taking behaviors including driving recklessly, which leads to increased incidence of MVA, more chances of unprovoked falls, and violence-related injuries [6].

Alcohol intoxication has a direct neurotoxic effect on the brain in patients with AUD. It causes damage to the frontal lobe, cerebellum and the limbic system. Alcohol intoxication can lead to widespread white matter cerebral atrophy due to neuronal degeneration. These brain changes lead to impairment in cognition and mood abilities such as poor judgment, a decrease in concentration, impaired emotional processing, goaldirected behavior, and balance problems [7]. TBI causes diffuse axonal injury in the white matter and cerebral hemispheres leading to a lack of coordination, dizziness, poor judgment, impaired cognition, and judgment [8]. So, the co-occurrence of TBI with AUD has augmented long-term adverse effects on the brain functions [9]. AUD can help in the short-term reduction of depressive symptoms in post-TBI patients; however, it has a long-term impact on worsening anxiety and depressive symptoms [10].

Adolescents with TBI are at high risk of developing psychiatric disorders and substance abuse. Anxiety disorders, major depressive disorder (MDD), attention deficit hyperactivity disorder (ADHD), bipolar disorder, obsessive-compulsive disorder (OCD), and post-traumatic stress disorder (PTSD) are common after TBI [11]. Comorbid psychiatric disorders and AUD after TBI have negative outcomes on cognition functions and recovery $[10,12]$.

We conducted a cross-sectional study to identify the demographic predictors of TBI, and the risk of association of psychiatric comorbidities including AUD and TBI-related hospitalizations in the children and adolescent population. Next, we discerned the differences in demographics, psychiatric comorbidities, and substance use disorders (SUDs) in TBI inpatients with AUD.

\section{Materials And Methods}

\section{Data source}

We performed a retrospective cross-sectional analysis of the nationwide inpatient sample (NIS) database (2010 to 2014) from the healthcare cost and utilization project (HCUP) [13]. The NIS provides discharge patient data from a $20 \%$ sample of all hospitals, and when discharge weights are applied to the data, the result is a weighted estimate of the total number of hospitalizations representing the US population [13]. Diagnostic information in the NIS is detected using the International Classification of Diseases, ninth edition (ICD-9) codes [13].

\section{Inclusion criteria and outcome variables}

We included children (age 8-18 years) with a primary ICD-9 discharge diagnosis for TBI as indicated by skull fractures (codes 800-801, 803-804); intracranial injury, including concussion, contusion, laceration, and hemorrhage (850.0-854.1); or unspecified head injury (959.01). Co-diagnosis of AUD was identified using the ICD-9 codes 291.0-291.3, 291.5, 291.8, 291.81, 281.82, 291.89, 291.9, 303.00-303.93 or 305.00-305.03.

Demographic variables studied included age, gender, and race [14]. The comorbid psychiatric illnesses included mood disorders, anxiety disorders, and psychotic disorders, and among SUD we included tobacco, cannabis, cocaine/amphetamine (stimulants), and opioids. These comorbidities were identified using ICD-9 diagnosis codes [14].

\section{Statistical analysis}

We compared non-TBI and TBI cohorts using the bivariate analysis to evaluate the differences in demographics and comorbidities, and then in the separate bivariate analysis, we compared TBI inpatients with AUD versus without AUD. A multivariable logistic regression model adjusted for demographics, psychiatric comorbidities, and SUD was used to evaluate the odds ratio (OR) of AUD as a risk factor for TBIrelated hospitalization. All statistical analyses set a priori at $<0.01$ were conducted on the Statistical Package for the Social Sciences (SPSS) version 25 (IBM Corp., Armonk, NY).

\section{Ethical approval}

Individual identifiers were used to protect the patient identity and other clinical information. The use of NIS under the HCUP does not require approval from the institutional review board as the NIS is a publicly available de-identified database [13].

\section{Results}

The majority of the TBI inpatients were adolescents (12-18 years, 82.2\%) and had higher odds (OR 1.46, 95\% CI 1.43-1.49) for hospitalization for TBI compared to children under 11 years. TBI was prevalent in males (71.2\% vs. 28.8\% females) and they had three times higher odds (95\% CI 3.14-3.26) for TBI-related hospitalization compared to females. TBI inpatients were seen majorly in whites (59.2\%) and there was a statistically significant difference race-wise with minorities at lower odds for TBI-related hospitalization. 


\section{Cureus}

Among psychiatric comorbidities, mood (4.1\%) and anxiety (2.2\%) disorders were prevalent in TBI inpatients, and when compared with non-TBI cohort, TBI inpatients have lower odds of association with psychiatric comorbidities. Among substance use disorders (SUDs), alcohol and tobacco use (4.4\% each), and cannabis use (3.5\%) were prevalent. SUD did not have a positive association with TBI-related hospitalization. AUD was associated with higher odds (OR 3.5, 95\% CI 3.35-3.67) of TBI-related hospitalization in the logistic regression model after adjusting for demographics, psychiatric comorbidities, and SUD as shown in Table 1.

\begin{tabular}{|c|c|c|c|c|c|}
\hline Variables & Non-TBI & TBI & Odds ratio & $95 \% \mathrm{Cl}$ & P-value \\
\hline Total inpatients & $3,763,575$ & 61,948 & - & - & - \\
\hline \multicolumn{6}{|l|}{ Age groups, \% } \\
\hline $8-11$ years & 20.3 & 17.8 & Reference & & \\
\hline 12-18 years & 79.7 & 82.2 & 1.46 & $1.43-1.49$ & $<0.01$ \\
\hline \multicolumn{6}{|l|}{ Sex, \% } \\
\hline Male & 43.4 & 71.2 & 3.19 & $3.14-3.26$ & $<0.01$ \\
\hline Female & 56.6 & 28.8 & Reference & & \\
\hline \multicolumn{6}{|l|}{ Race, \% } \\
\hline Caucasian & 51.6 & 59.2 & Reference & & \\
\hline African American & 20.1 & 14.3 & 0.62 & $0.61-0.64$ & $<0.01$ \\
\hline Hispanic & 20.8 & 18.8 & 0.79 & $0.77-0.81$ & $<0.01$ \\
\hline Other & 7.5 & 7.8 & 0.89 & $0.87-0.92$ & $<0.01$ \\
\hline \multicolumn{6}{|c|}{ Psychiatric comorbidities, in \% } \\
\hline No comorbidities & - & - & Reference & & \\
\hline Anxiety disorders & 8.8 & 2.2 & 0.28 & $0.27-0.29$ & $<0.01$ \\
\hline Mood disorders & 8.5 & 4.1 & 0.52 & $0.50-0.55$ & $<0.01$ \\
\hline Psychotic disorders & 0.9 & 0.6 & 0.72 & $0.64-0.81$ & $<0.01$ \\
\hline \multicolumn{6}{|c|}{ Substance use disorders, in \% } \\
\hline Alcohol & 1.8 & 4.4 & 3.50 & $3.35-3.67$ & $<0.01$ \\
\hline Tobacco & 4.0 & 4.4 & 0.98 & $0.94-1.02$ & 0.318 \\
\hline Cannabis & 4.2 & 3.5 & 0.59 & $0.57-0.63$ & $<0.01$ \\
\hline Opioid & 0.6 & 0.2 & 0.35 & $0.29-0.42$ & $<0.01$ \\
\hline Stimulants & 0.6 & 0.5 & 0.85 & $0.75-0.96$ & 0.01 \\
\hline
\end{tabular}

TABLE 1: Predictors for traumatic brain injury-related hospitalization

$\mathrm{TBI}$, traumatic brain injury

TBI inpatients with AUD were majorly adolescents (96.4\%) who had four times higher odds (95\% CI 3.46$5.21)$ compared to children under 11 years. AUD was prevalent in males (77.2\%) and they had higher odds (OR 1.17, 95\% CI 1.06-1.29) than females. Though AUD was prevalent in Caucasians (53.3\%), yet compared with them, hispanics had 1.9 times higher odds (95\% CI 1.72-2.09) for AUD.

Statistically there was no significant difference for anxiety and psychotic disorders by comorbid AUD. TBI inpatients with AUD had higher odds (OR 1.62, 95\% CI 1.37-1.92) for mood disorders. Among SUD, TBI inpatients with AUD had higher odds for comorbid stimulants (OR 5.11, 95\% CI 3.85-6.77), cannabis (OR 4.69, 95\% CI 4.12-5.34), and tobacco (OR 3.77, 95\% CI 3.34-4.27) as shown in Table 2. 


\section{Cureus}

\begin{tabular}{|c|c|c|c|c|c|}
\hline Variables & Non-AUD & AUD & Odds ratio & $95 \% \mathrm{Cl}$ & P-value \\
\hline Total inpatients & 59,206 & 2,644 & - & - & - \\
\hline \multicolumn{6}{|l|}{ Age groups, \% } \\
\hline 8-11 years & 18.5 & 3.6 & Reference & & \\
\hline 12-18 years & 81.5 & 96.4 & 4.24 & $3.46-5.21$ & $<0.01$ \\
\hline \multicolumn{6}{|l|}{ Sex, \% } \\
\hline Male & 70.9 & 77.2 & 1.17 & $1.06-1.29$ & $<0.01$ \\
\hline Female & 29.1 & 22.8 & Reference & & \\
\hline \multicolumn{6}{|l|}{ Race, \% } \\
\hline Caucasian & 59.4 & 53.3 & Reference & & \\
\hline African American & 14.7 & 6.0 & 0.41 & $0.35-0.49$ & $<0.01$ \\
\hline Hispanic & 18.2 & 31.3 & 1.89 & $1.72-2.09$ & $<0.01$ \\
\hline Other & 7.7 & 9.3 & 1.37 & 1.18-1.59 & $<0.01$ \\
\hline \multicolumn{6}{|c|}{ Psychiatric comorbidities, in \% } \\
\hline No comorbidities & - & - & Reference & & \\
\hline Anxiety disorders & 2.2 & 3.2 & 0.71 & $0.54-0.93$ & 0.014 \\
\hline Mood disorders & 3.9 & 8.5 & 1.62 & 1.37-1.92 & $<0.01$ \\
\hline Psychotic disorders & 0.5 & 1.3 & 1.14 & $0.75-1.73$ & 0.552 \\
\hline \multicolumn{6}{|c|}{ Substance use disorders, in \% } \\
\hline Tobacco & 3.7 & 20.5 & 3.77 & $3.34-4.27$ & $<0.01$ \\
\hline Cannabis & 2.6 & 21.4 & 4.69 & $4.12-5.34$ & $<0.01$ \\
\hline Opioid & 0.2 & 0.4 & 0.35 & $0.16-0.77$ & 0.01 \\
\hline Stimulants & 0.3 & 4.3 & 5.11 & $3.85-6.77$ & $<0.01$ \\
\hline
\end{tabular}

\section{TABLE 2: Characteristics of traumatic brain injury inpatients with comorbid alcohol use disorder}

AUD, alcohol use disorder

\section{Discussion}

The results of our large sample cross-sectional nationwide study found that AUD increases the risk for TBIrelated hospitalization in the children and adolescent population by 3.5 times. AUD is an independent risk factor for TBI as the regression model was adjusted for potential confounders including SUD and psychiatric comorbidities.

TBI hospitalizations are more common in boys compared to girls among adolescents. This is probably due to an increased incidence of TBI due to MVA and/or intentional assault, which is common among boys [15]. In our study, adolescents (increased by 1.5 times) and males (increased by three times) had higher odds for TBIrelated hospitalization. TBI is more common in blacks compared to other races due to the fact that high violence rates are seen in black teens [16]. Black children are significantly less likely to have ED visits after a head injury, and less likely to be diagnosed with brain concussion compared to whites [17]. According to a study on children and adolescent hospitalizations, most children with TBI are male and white [18]. This explains a higher prevalence of TBI-related hospitalization in whites compared to blacks and other races in our study.

Depression is common in post-TBI patients due to difficulty coping with the loss in the individual's life [19]. MDD occurs in 10\%-25\% of post-TBI children [20]. TBI causes disruption in the neuronal circuit, which causes disruptions of the neurotransmitter circuits in serotonin, norepinephrine, dopamine, and acetylcholine leading to a higher risk of anxiety and mood disorders [21]. In our study, none of the 
Adolescents are at high risk of addiction due to the continued brain and/or grey matter maturation and neurodevelopmental changes that take place in the frontal cortical and the subcortical monoaminergic systems. These changes explain the adolescents impulsive and novelty-seeking behavior traits [22]. TBI causes disruption to the developing nervous system, which increases the vulnerability to AUD and SUD [23]. TBI increases the risk of addiction by reducing the cognitive ability to perceive negative consequences and increases the perception of gains perceived by drinking and substance abuse [23]. A study by Patel et al. found that cannabis abuse among youth increases the risk of intracranial injury by 1.3 times [24]. Binge alcohol drinking is also a risk factor for TBI due to falls and assaults and MVA. After controlling for demographic confounders, psychiatric comorbidities, and other SUD, AUD was associated with statistically significant increased risk for TBI-related hospitalization.

AUD is more common in boys compared to girls, due to biological and social reasons. The increase in dopamine in the ventral striatum, more specifically nucleus accumbens, in boys is responsible for pleasure, reinforcement, and development of SUD [25]. Both sex face different social reinforcement and punishment attitudes regarding alcohol use. Alcohol use for boys is perceived as a sign of masculinity and independence in certain cultures, whereas among girls, it is expected to embrace the cultural stereotypical female characteristics like virtue and emotionality, consequently, they consume less alcohol [26]. In our TBI inpatients, AUD was seen majorly in males (77\%) and adolescents (increased by four times compared to children $<11$ years).

Among those who drink alcohol, hispanics are at higher risk of AUD compared to non-hispanic whites [27]. This correlates with our study findings, as 31.3\% hispanics had about two times higher odds for AUD than $53.3 \%$ whites. Alcohol, cannabis, and tobacco are the most common substances abused among adolescents in the United States, with prevalence rates $24 \%, 13 \%, 13 \%$, respectively [28]. The co-occurrence of cannabis and tobacco use with alcohol is very common in adolescents. About $75 \%$ of American teens who use cannabis also use alcohol. The use of more than one substance increases the likelihood of more than one SUD [29]. According to the substance abuse and mental health services administration (SAMSHA), 53\% of adolescents who are binge drinking, reported last month tobacco use, and $30 \%$ of binge drinkers and $56 \%$ of tobacco users reported cannabis use in the same period [28]. In our study, we found that TBI inpatients with AUD had four to five times higher odds for comorbid tobacco and cannabis use disorders. Alcohol and methamphetamine are frequently used together, and about $77 \%$ of those who have comorbid methamphetamine use disorders also meet the criteria for AUD. AUD increases the chances of methamphetamine use by four-folds for an increased euphoric effect [30]. This could be the possible reason behind TBI inpatients with AUD having five times higher odds for abusing stimulants.

An important limitation of our study is that the NIS data lack individual-level clinical information and the cross-sectional nature of the study prevents from confirming the causal relationship between TBI and AUD in hospitalized adolescents. But, using these national data, we were able to confirm a population-based association of AUD and TBI and an increase in hospitalizations in adolescents after controlling for possible confounder.

\section{Conclusions}

AUD is an independent risk factor for TBI-related hospitalization with an increased risk of $50 \%$ in the children and adolescent population compared to non-alcohol users. TBI inpatients with AUD are prevalent in white, and male adolescents. These at-risk populations are also at higher risk of comorbid mood disorders and increased substance use including stimulants, cannabis, and tobacco. Alcohol screening and support groups, and behavioral health education programs should be provided to post-TBI patients with AUD as persistent alcohol use problems leads to cognitive deficits, worsening memory, learning abilities, and processing speed. They also have less tolerance to alcohol and more liability to falls, seizures, and subsequent TBI with low blood alcohol levels.

\section{Additional Information \\ Disclosures}

Human subjects: Consent was obtained by all participants in this study. Animal subjects: All authors have confirmed that this study did not involve animal subjects or tissue. Conflicts of interest: In compliance with the ICMJE uniform disclosure form, all authors declare the following: Payment/services info: All authors have declared that no financial support was received from any organization for the submitted work. Financial relationships: All authors have declared that they have no financial relationships at present or within the previous three years with any organizations that might have an interest in the submitted work. Other relationships: All authors have declared that there are no other relationships or activities that could appear to have influenced the submitted work.

\section{References}

1. Pediatric traumatic brain injury. (2020). Accessed: May 10, 2020: 
https://www.asha.org/PRPSpecificTopic.aspx?folderid=8589942939\&section=Incidence_and_Prevalence.

2. Thurman DJ: The epidemiology of traumatic brain injury in children and youths: a review of research since 1990. J Child Neurol. 2016, 31:20-27. 10.1177/0883073814544363

3. Langlois JA, Rutland-Brown W, Thomas KE: The incidence of traumatic brain injury among children in the United States: differences by race. J Head Trauma Rehabil. 2005, 20:229-238. 10.1097/00001199-20050500000006

4. Alcohol facts and statistics. (2020). Accessed: May 10, 2020:

https://www.niaaa.nih.gov/publications/brochures-and-fact-sheets/alcohol-facts-and-statistics.

5. Weil ZM, Corrigan JD, Karelina K: Alcohol use disorder and traumatic brain injury . Alcohol Res. 2018, 39:171-180.

6. Substance use/abuse and TBI. (2012). Accessed: May 10, 2020: http://braininjuryeducation.org/TBIBasics/Substance-Abuse-and-TBI/.

7. Oscar-Berman M, Marinkovic K: Alcohol: effects on neurobehavioral functions and the brain . Neuropsychol Rev. 2007, 17:239-257. 10.1007/s11065-007-9038-6

8. Kraus MF, Susmaras T, Caughlin BP, Walker CJ, Sweeney JA, Little DM: White matter integrity and cognition in chronic traumatic brain injury: a diffusion tensor imaging study. Brain. 2007, 130:2508-2519. 10.1093/brain/awm216

9. Pagulayan KF, Temkin NR, Machamer JE, Dikmen SS: Patterns of alcohol use after traumatic brain injury . J Neurotrauma. 2016, 33:1390-1396. 10.1089/neu.2015.4071

10. Herrold AA, Sander AM, Wilson KV, Scimeca LM, Cobia DJ, Breiter HC: Dual diagnosis of traumatic brain injury and alcohol use disorder: characterizing clinical and neurobiological underpinnings. Curr Addict Rep. 2015, 2:273-284. 10.1007/s40429-015-0078-3

11. Whelan-Goodinson R, Ponsford J, Johnston L, Grant F: Psychiatric disorders following traumatic brain injury: their nature and frequency. J Head Trauma Rehabil. 2009, 24:324-332. 10.1097/HTR.0b013e3181a712aa

12. Jorge RE, Robinson RG, Moser D, Tateno A, Crespo-Facorro B, Arndt S: Major depression following traumatic brain injury. Arch Gen Psychiatry. 2004, 61:42-50. 10.1001/archpsyc.61.1.42

13. Overview of the national (nationwide) inpatient sample . (2019). Accessed: March 15, 2020: https://www.hcup-us.ahrq.gov/nisoverview.jsp.

14. NIS description of data elements . (2019). Accessed: March 15, 2020: https://www.hcupus.ahrq.gov/db/nation/nis/nisdde.jsp.

15. Collins NC, Molcho M, Carney P, McEvoy L, Geoghegan L, Phillips JP, Nicholson AJ: Are boys and girls that different? an analysis of traumatic brain injury in children. Emerg Med J. 2013, 30:675-678. 10.1136/emermed-2011-200496

16. Report to congress: traumatic brain injury in the United States . (2016). Accessed: May 5, 2020: https://www.cdc.gov/traumaticbraininjury/pubs/tbi_report_to_congress.html.

17. Lyons TW, Miller KA, Miller AF, Mannix R: Racial and ethnic differences in emergency department utilization and diagnosis for sports-related head injuries. Front Neurol. 2019, 10:690. 10.3389/fneur.2019.00690

18. Reuter-Rice K, Doser K, Eads JK, Berndt S: Pediatric traumatic brain injury: families and healthcare team interaction trajectories during acute hospitalization. J Pediatr Nurs. 2017, 34:84-89. 10.1016/j.pedn.2016.12.017

19. Emotional problems after traumatic brain injury . (2019). Accessed: May 5, 2020: https://www.brainline.org/article/emotional-problems-after-traumatic-brain-injury.

20. Morse AM, Garner DR: Traumatic brain injury, sleep disorders, and psychiatric disorders: an underrecognized relationship. Med Sci. 2018, 6:15. 10.3390/medsci6010015

21. van Reekum R, Cohen T, Wong J: Can traumatic brain injury cause psychiatric disorders? . J Neuropsychiatry Clin Neurosci. 2000, 12:316-327.

22. Chambers RA, Taylor JR, Potenza MN: Developmental neurocircuitry of motivation in adolescence: a critical period of addiction vulnerability. Am J Psychiatry. 2003, 160:1041-1052. 10.1176/appi.ajp.160.6.1041

23. Weil ZM, Karelina K: Traumatic brain injuries during development: implications for alcohol abuse . Front Behav Neurosci. 2017, 11:135. 10.3389/fnbeh.2017.00135

24. Patel RS, Mekala HM, Tankersley WE: Cannabis use disorder and epilepsy: a cross-national analysis of 657 072 hospitalized patients. Am J Addict. 2019, 28:353-360. 10.1111/ajad.12898

25. Urban NB, Kegeles LS, Slifstein M, et al.: Sex differences in striatal dopamine release in young adults after oral alcohol challenge: a positron emission tomography imaging study with [ $\left.{ }^{11} \mathrm{c}\right]$ raclopride. Biol Psychiatry. 2010, 68:689-696. 10.1016/j.biopsych.2010.06.005

26. Schulte MT, Ramo D, Brown SA: Gender differences in factors influencing alcohol use and drinking progression among adolescents. Clin Psychol Rev. 2009, 29:535-547. 10.1016/j.cpr.2009.06.003

27. Alcohol and the hispanic community. (2019). Accessed: May 5, 2020: https://www.niaaa.nih.gov/publications/brochures-and-fact-sheets/alcohol-and-hispanic-community.

28. Banks DE, Rowe AT, Mpofu P, Zapolski TCB: Trends in typologies of concurrent alcohol, marijuana, and cigarette use among us adolescents: an ecological examination by sex and race/ethnicity. Drug Alcohol Depend. 2017, 179:71-77. 10.1016/j.drugalcdep.2017.06.026

29. Schlienz NJ, Lee DC: Co-use of cannabis, tobacco, and alcohol during adolescence: policy and regulatory implications. Int Rev Psychiatry. 2018, 30:226-237. 10.1080/09540261.2018.1465399

30. Singh AK: Alcohol interaction with cocaine, methamphetamine, opioids, nicotine, cannabis, and gammahydroxybutyric acid. Biomedicines. 2019, 7:16. 10.3390/biomedicines7010016 